\title{
Adjuvant Capecitabine-Containing Chemotherapy Benefit and Homologous Recombination Deficiency Status Among Early-Stage TNBC Patients in the FinXX trial
}

\section{Leonora W. de Boo}

Netherlands Cancer Institute: Antoni van Leeuwenhoek Nederlands Kanker Instituut

Katarzyna Jóźwiak

Brandenburg medical school Theodor Fontane

Heikki Joensuu

Helsinki University Central Hospital Department of Oncology: Syopatautien klinikka Henrik Lindman

Uppsala University Hospital: Akademiska sjukhuset

\section{Susanna Lauttia}

University of Helsinki City Centre Campus: Helsingin Yliopisto

\section{Mark Opdam}

Netherlands Cancer Institute: Antoni van Leeuwenhoek Nederlands Kanker Instituut

\section{Charlaine van Steenis}

Netherlands Cancer Institute: Antoni van Leeuwenhoek Nederlands Kanker Instituut

\section{Wim Brugman}

Netherlands Cancer Institute: Antoni van Leeuwenhoek Nederlands Kanker Instituut

Roelof J.C. Kluin

Netherlands Cancer Institute: Antoni van Leeuwenhoek Nederlands Kanker Instituut

\section{Philip C. Schouten}

Netherlands Cancer Institute: Antoni van Leeuwenhoek Nederlands Kanker Instituut

\section{Marleen Kok}

Netherlands Cancer Institute: Antoni van Leeuwenhoek Nederlands Kanker Instituut

\section{Petra M. Nederlof}

Netherlands Cancer Institute: Antoni van Leeuwenhoek Nederlands Kanker Instituut

\section{Michael Hauptmann}

Brandenburg Medical School Theodor Fontane: Medizinische Hochschule Brandenburg Theodor Fontane

\section{Sabine Linn ( $\sim$ s.linn@nki.nl)}

Netherlands Cancer Institute https://orcid.org/0000-0001-5541-0347 


\section{Research article}

Keywords: BRCA1 protein/genetics, Homologous Recombination Deficiency, Breast cancer, Triple-negative Breast Neoplasms, Capecitabine, Adjuvant chemotherapy, Predictive biomarker

Posted Date: December 11th, 2020

DOl: https://doi.org/10.21203/rs.3.rs-125140/v1

License: (c) (i) This work is licensed under a Creative Commons Attribution 4.0 International License.

Read Full License

Version of Record: A version of this preprint was published at British Journal of Cancer on January 17th, 2022. See the published version at https://doi.org/10.1038/s41416-022-01711-y. 


\section{Abstract}

Background: Recent data demonstrate that patients with early-stage triple negative breast cancer (TNBC) benefit from escalating adjuvant treatment with capecitabine. However, since a substantial proportion of patients does not benefit, predictive biomarkers to select those individuals upfront are needed. Over half of all TNBCs have a BRCA7-like DNA copy number signature similar to the profile found in germline BRCA1-mutated breast cancers and indicative for homologous recombination deficiency. We evaluate this signature as a predictive biomarker for capecitabine benefit in archived specimens of the randomized controlled FinXX trial. Additionally, we compared the concordance of our DNA-based BRCA7-like classifier with the RNA-based NanoString BRCAness signature.

Methods: Early-stage TNBC patients were randomized between adjuvant capecitabine-containing chemotherapy ( $T X+C E X$ : capecitabine plus docetaxel, followed by cyclophosphamide, epirubicin and capecitabine) and conventional adjuvant chemotherapy (T+CEF: docetaxel, followed by cyclophosphamide, epirubicin, and fluorouracil). Breast tumor BRCA1-like status was determined on low coverage, whole genome next-generation sequencing data using an established DNA comparative genomic hybridization algorithm. We used interaction analysis in proportional hazards models to evaluate whether benefit of adjuvant capecitabine-containing versus conventional chemotherapy differs between $B R C A 7$-like and non-BRCA1-like tumors in early-stage TNBC patients.

Results: For 129 (63.9\%) of the 202 TNBC patients the BRCA7-like status could be determined. Thirty-five recurrences and 32 deaths occurred during a median follow-up of 10.7 years. The capecitabine effect on recurrence-free survival did not significantly differ between the 68 patients $(52.7 \%)$ with a BRCA7-like tumor (HR 0.66, 95\% Cl 0.24-1.81) and others (HR 0.23, 95\% Cl 0.08-0.70, P interaction $=0.17$ ), also after adjustment for clinico-pathological variables.

Conclusions: In the FinXX trial, the BRCA7-like status was not associated with a differential benefit from capecitabine-containing adjuvant chemotherapy compared to conventional chemotherapy in the TNBC subgroup. Based on this study, it is unlikely that the BRCA7-like classifier can be used to distinguish patients who do and do not benefit from capecitabine-enriched standard adjuvant chemotherapy.

\section{Background}

Triple negative breast cancer (TNBC) accounts for $10-20 \%$ of all breast cancers and is associated with a high risk of early recurrence and poor survival once metastasized [1, 2]. Trials evaluating escalation of adjuvant treatment are emerging $[3,4]$, including trials on the addition of capecitabine for early-stage TNBC patients. This prodrug of 5-fluoruouracil belongs to the class of antimetabolites and shows cytotoxic activity through the inhibition of thymidylate synthase and the incorporation of its metabolites into DNA and RNA [5]. A recent meta-analysis including 3,854 early-stage TNBC patients showed that adjuvant capecitabine following or added to standard neoadjuvant anthracycline- and taxane-based therapy substantially improved disease-free survival (DFS) and overall survival (OS) $[6,7]$. Hence, this 
approach has been incorporated in national and international guidelines [8-10]. Although these results are promising, a substantial proportion of TNBC patients does not benefit from the addition of adjuvant capecitabine. This is, for instance, illustrated by the $8.5 \%$ absolute overall survival benefit at 5 -years for TNBC patients who did not achieve a pathological complete remission on neoadjuvant chemotherapy with subsequently 6-8 courses of capecitabine versus those who did not receive capecitabine $(78.8 \%$ versus $70.3 \%$, respectively) [7]. To our knowledge, the only study exploring biomarkers that predict capecitabine benefit failed to identify a predictive marker in an 800-gene expression analysis [11]. Therefore, it remains unclear which early-stage TNBC patients have a high chance of benefitting from capecitabine.

Homologous recombination deficiency (HRD) may serve as a putative predictive biomarker to guide decisions on systemic therapy for patients with early-stage TNBC, especially for DNA damaging agents $[12,13]$. In unselected TNBC patients, approximately $10 \%$ of the patients harbor a deleterious $B R C A 1 / 2$ mutation which results in tumors that are deficient in homologous recombination [14-18]. In TNBC patients without a germline $B R C A 1 / 2$ mutation, a significant number of tumors harbor $\operatorname{HRD}[12,19,20]$. The array comparative genomic hybridization (aCGH) BRCA7-like classifier is an HRD-test that has been developed from the characteristic DNA copy number aberrations of BRCA7-mutated breast cancers [21]. This BRCA1-like classifier showed clinical validity and utility to predict benefit of intensified platinumbased chemotherapy for stage III HER2-negative breast cancer patients [22-25]. However, the predictive value of the BRCA7-like classifier for outcome after (neo)adjuvant treatment with other DNA damaging agent-containing regimens and/or dose-intensities is currently unknown.

In data from two studies contributing to the results of the meta-analysis of Van Mackelenbergh et al. [6], the GAIN trial and the CREATE-X trial, there are indications that patients with BRCA1-like TNBC tumors may benefit from capecitabine-containing treatment. First, Van Rossum et al. observed a trend for improved survival in the BRCA7-like TNBC patients of the GAIN trial treated with capecitabine-containing chemotherapy compared with intensified dose-dense chemotherapy [26]. Second, based on the CREATE-X trial, which was limited to patients with residual disease after neoadjuvant treatment, it seems that capecitabine could have a greater role in patients with tumors that are less sensitive or partially resistant to regimens containing anthracyclines and taxanes [7]. We hypothesize that the improved outcome of TNBC patients treated with capecitabine-containing chemotherapy in the CREATE-X and the FinXX trial were driven by patients with BRCA1-like tumors. Contrarily, recent findings of Asleh et al., using a RNAbased 800-gene panel in exploratory analyses, suggest that the BRCAness signature is not associated with the benefit of adjuvant capecitabine in early-stage TNBC [11]. Therefore, the predictive value of the DNA-based BRCA7-like status for the differential benefit from adjuvant capecitabine in early-stage TNBC needs further evaluation.

Our aim is to evaluate whether $B R C A 7$-like status determines benefit of adjuvant capecitabine-containing systemic treatment in early-stage TNBC patients within the FinXX trial. The FinXX trial is a large phase III, randomized controlled trial comparing adjuvant conventional chemotherapy with adjuvant capecitabinecontaining chemotherapy [27]. 


\section{Methods}

\section{Patients}

We studied early-stage TNBC patients who were included in the Finland Capecitabine (FinXX) trial; a large, multicenter, randomized controlled clinical trial conducted in Finland and Sweden between 2004 and 2007 [27, 28]. Eligibility criteria have been published previously [27]. In summary, the patients were younger than 65 years, had histologically confirmed invasive breast cancer with either regional lymph nodes containing cancer or node negative cancer with primary tumors of $\geq 20 \mathrm{~mm}$ diameter and negative progesterone receptor (PR) expression in immunohistochemistry, no distant metastases, and no prior neoadjuvant chemotherapy. TNBC was defined as estrogen (ER) and progesterone receptor (PR) negativity (<10\%), and no HER2 overexpression (determined either by immunohistochemistry or in situ hybridization). The study was approved by the Ethics Committee of the participating medical institutions and the National Agency for Medicines, Finland. Patients supplied written informed consent to allow the use of their tumor tissue for clinical study related research purposes. The Institutional Review Board at the Helsinki University Hospital, Finland, approved the use of archival tissue for the current translational study.

\section{Treatment}

Patients were randomized in a 1:1 ratio to either an adjuvant capecitabine $(X)$-containing chemotherapy regimen (TX + CEX: 3 cycles of capecitabine $900 \mathrm{mg} / \mathrm{m}^{2}$ twice daily plus docetaxel $60 \mathrm{mg} / \mathrm{m}^{2}$ 3-weekly, followed by 3 cycles of cyclophosphamide $600 \mathrm{mg} / \mathrm{m}^{2}$, epirubicin $75 \mathrm{mg} / \mathrm{m}^{2}$ and capecitabine $900 \mathrm{mg} / \mathrm{m}^{2}$ twice daily, 3-weekly) or to adjuvant conventional chemotherapy ( $\mathrm{T}+\mathrm{CEF}: 3$ cycles of docetaxel $80 \mathrm{mg} / \mathrm{m}^{2}$ 3-weekly, followed by 3 cycles of cyclophosphamide $600 \mathrm{mg} / \mathrm{m}^{2}$, epirubicin $75 \mathrm{mg} / \mathrm{m}^{2}$, and fluorouracil $600 \mathrm{mg} / \mathrm{m}^{2}$, 3-weekly). Patients received locoregional radiotherapy after completion of chemotherapy according to the local guidelines.

\section{DNA extraction}

Tumor DNA was isolated from two $10 \mu \mathrm{m}$ whole slides of formalin-fixed paraffin-embedded (FFPE) tissue containing at least $50 \%$ tumor cells. A manual microdissection was carried out for slides containing $\leq$ $50 \%$ of representative tumor area to increase the percentage of neoplastic cells. Paraffin was removed with Qiagen's Deparaffinization Solution, and tissue was lysed using a mixture of $20 \mu \mathrm{L}$ Proteinase K (20 mg/ml, included in the QIAsymphony DSP DNA kit) and $200 \mu \mathrm{L}$ lysis buffer $(0.05 \mathrm{M}$ Tris-HCl ph 8.5 , $0.04 \mathrm{mM}$ EDTA, $0.5 \%$ Tween 20) per sample at $56^{\circ} \mathrm{C}$ overnight. DNA extraction was performed with QIAsymphony SP instrument using DSP DNA mini kit with $100 \mu \mathrm{L}$ elution volume (Qiagen, Venlo, The Netherlands).

\section{Low coverage whole genome sequencing and data processing}


The amount of double-stranded DNA in the genomic DNA samples was quantified using the Qubit ${ }^{\circledR}$ dsDNA HS Assay Kit (Invitrogen, cat no Q32851). Up to 500 ng of double-stranded genomic DNA was fragmented using ultrasonicator shearing (Covaris.com, Massachusetts, USA) to obtain fragment sizes of 160-180 bp. Samples were purified using 1.8X Agencourt AMPure XP PCR Purification beads according to manufacturer's instructions (Beckman Coulter, cat no A63881). DNA library preparation for Illumina sequencing was performed using the KAPA Hyper Prep Kit (KAPA Biosystems, KK8504). During the ligation 144 unique adapter indices, manufactured by IDT (Integrated DNA Technologies IDT, Inc. Coralville, lowa, USA), were used in a molarity of $15 \mu \mathrm{M}$. Six PCR cycles were used during library enrichment to obtain enough yield for sequencing. All DNA libraries were analyzed on the Caliper GX bioanalyzer (PerkinElmer) using the HT DNA High Sensitivity LabChip for determining the molarity. Up to 133 uniquely indexed samples were mixed together by equimolar pooling. The pools were analyzed on the Agilent Technologies 2100 Bioanalyzer and subsequently diluted to $10 \mathrm{nM}$. Each pool was subjected to sequencing in one lane of a single read 65 bp run, on an Illumina HiSeq2500 machine, according to manufacturer's instructions.

Reads were aligned to the reference genome GRCh38 using BWA-MEM algorithm (version 0.7.17) [29]. Per bin of $20 \mathrm{~kb}$, using BEDTools [30], reads on autosomes were counted. Excluded were sites attracting excessive anomalous read mappings (ENCODE) [31] and bins that had a GRCh38 reference mappability below 0.2. Mappability is the fraction of 65 bp sequences, per bin, that aligns to itself. Local GC effects in samples were fitted with a non-linear loess, including a subset of reference mappabilities over 0.8 , to correct sample bin counts. A line can be fitted through the origin and center of GC corrected counts per mappability density. The slope of this line is used to scale mappabilities to reference counts. Genomic profiles consist of log2 ratios of GC corrected bin counts divided by these scaled reference counts.

The sequencing data discussed in this publication have been deposited in NCBI's Sequence Read Archive (SRA) and are accessible through BioProject number PRJNA647428 [32].

\section{BRCA1 -like classification}

Genomic profiles were analyzed using a BRCA1-like classifier, which was originally developed using array comparative genomic hybridization $(\mathrm{aCGH})$ data generated from breast cancers that were or were not associated with germline BRCA1 mutations [21]. In brief, the BRCA1-like classifier is a shrunken centroid classifier that assigns a genomic profile to a $B R C A 7$-like class using a probability score between 0 (non$B R C A 1$-like) and 1 (BRCA1-like). The threshold for assigning a breast tumor to the BRCA1-like group was set at $\geq 0.63$ as obtained and validated in previous studies [22-25]. The BRCA7-like classifier can be used on genomic copy number variation (CNV) profiles obtained by low coverage whole genome sequencing $[26,33]$. Recently, we implemented several updates in the processing of CNV sequencing (CNVseq) data and validated the BRCA1-like classification obtained with these data. A detailed description is provided in the Supplementary methods (Additional file 1: Supplementary methods). In brief, the BRCA1-like classification of copy number profiles can reliably be obtained with the updated CNVseq data with an accuracy of $85-93 \%$ when compared to the original BAC aCGH BRCA7-like 
classifier (which is similar to previously established performance on low coverage, whole genome nextgeneration sequencing [33]).

Quality checks of the CNV profiles of the TNBC FinXX patients were performed blinded for BRCA7-like score and outcome. Samples with low quality were excluded from analyses.

Previously, the identification of BRCAness has been explored on the same dataset of early-stage TNBC patients using the RNA-based NanoString BRCAness signature [11]. Signature scores were calculated using prescribed algorithms developed by NanoString technologies [34]. In the present study, we additionally compared the concordance of our DNA-based CNV BRCA1-like classifier with the RNA-based NanoString BRCAness signature.

\section{Statistical analyses}

Characteristics of patients were compared by BRCA7-like status using Fisher's exact, Chi-square or linearby-linear tests for categorical variables and Mann-Whitney $U$ tests for continuous variables.

Recurrence-free survival (RFS) was defined as time from randomization to local or distant invasive breast cancer recurrence, death from any cause, or to the last date of follow-up, whichever occurred first. Overall survival (OS) was defined as time from randomization to death from any cause or the last date of followup. Median follow-up was calculated using the reversed Kaplan Meier estimator. Survival curves were computed with the Kaplan-Meier method. To evaluate whether benefit from adjuvant capecitabinecontaining chemotherapy versus adjuvant conventional chemotherapy differs between BRCA7-like and non-BRCA7-like tumors, we applied Cox proportional hazards regressions that included the interaction term between treatment and BRCA1-like status. We estimated and compared interaction coefficients that were unadjusted and adjusted for the following variables: age at randomization, World Health Organization (WHO) performance status $(0,1)$, type of surgery (breast conserving, mastectomy), axillary surgery (dissection, sentinel node biopsy), T-stage (pT1, pT2, pT3), axillary nodal status ( $\leq 3$ vs $>3$ positive lymph nodes), histological type (ductal, lobular, other), and histological grade $(1,2,3)$. Due to the relatively small number of events, interaction coefficients were adjusted for one covariate at a time. The prognostic effects of all covariates were also evaluated in separate models. The proportionality of hazards was checked using Schoenfeld residuals. A two-sided p-value $<0.05$ was considered statistically significant. Statistical analyses were performed using SPSS version 25 (IBM Corp., Armonk, NY, USA) and Stata 16 (StataCorp., College Station, TX, USA).

To determine the concordance between our DNA-based CNV BRCA7-like classifier and the RNA-based NanoString BRCAness signature, we dichotomized the acquired continuous scores at the percentile of the established cut-off for the BRCA7-like classifier, as there is no predefined cut-off for the NanoString BRCAness signature.

\section{Results}




\section{Patient characteristics}

Of 202 TNBC patients included in the FinXX trial, we obtained BRCA7-like status for 129 (63.9\%) patients (Fig. 1). Main reasons for failure were lack of available tumor tissue, low tumor percentage and insufficient amounts of isolated DNA. This subgroup did not differ substantially for the variables mentioned in Table 1 from those TNBC FinXX patients not included in the current analyses (Additional file 2: Table S1). Sixty-eight (52.7\%) of the 129 tumors had a BRCA1-like profile (Table 1). As expected, patients with a BRCA7-like tumor had more frequently poorly differentiated tumors compared to patients with non-BRCA7-like tumors $(P=0.03)$ and had significantly more often less than four positive axillary lymph nodes $(P=0.047)$. Furthermore, BRCA1-like tumors had a higher T-stage $(P=0.03)$. 
Table 1

Characteristics of TNBC patients with known BRCA1-like status

\begin{tabular}{|c|c|c|c|c|c|c|c|}
\hline \multirow[t]{2}{*}{ Characteristic } & \multicolumn{2}{|c|}{ Total } & \multicolumn{2}{|c|}{$\begin{array}{l}\text { Patients with a } \\
\text { BRCA7-like profile }\end{array}$} & \multicolumn{2}{|c|}{$\begin{array}{l}\text { Patients with a non- } \\
\text { BRCA7-like profile }\end{array}$} & \multirow[t]{2}{*}{$\begin{array}{l}P \\
\text { value }\end{array}$} \\
\hline & $\mathrm{N}$ & (\%) & $\mathrm{N}$ & (\%) & $\mathrm{N}$ & (\%) & \\
\hline Total & 129 & $(100)$ & 68 & $(52.7)$ & 61 & $(47.3)$ & \\
\hline $\begin{array}{l}\text { Median (IQR) age at } \\
\text { study entry, y }\end{array}$ & 53 & $\begin{array}{l}(45- \\
59)\end{array}$ & 52 & $(44-58)$ & 54 & $(48-60)$ & 0.11 \\
\hline $\begin{array}{l}\text { WHO performance } \\
\text { status }\end{array}$ & & & & & & & 0.82 \\
\hline 0 & 109 & $(84.5)$ & 57 & (83.8) & 52 & $(85.2)$ & \\
\hline 1 & 20 & $(15.5)$ & 11 & $(16.2)$ & 9 & $(14.8)$ & \\
\hline $\begin{array}{l}\text { Median (IQR) tumor } \\
\text { diameter, mm }\end{array}$ & 25 & $\begin{array}{l}(21- \\
35)\end{array}$ & 28 & $(22-35)$ & 25 & $(19-35)$ & 0.07 \\
\hline T-stage & & & & & & & 0.03 \\
\hline pT1 & 32 & $(24.8)$ & 11 & $(16.2)$ & 21 & $(34.4)$ & \\
\hline pT2 & 87 & $(67.4)$ & 51 & $(75.0)$ & 36 & $(59.0)$ & \\
\hline рT3 & 10 & (7.8) & 6 & (8.8) & 4 & $(6.6)$ & \\
\hline Histological grade & & & & & & & 0.03 \\
\hline 1 & 1 & $(0.8)$ & 0 & (0) & 1 & $(1.6)$ & \\
\hline 2 & 15 & $(11.6)$ & 4 & $(5.9)$ & 11 & (18.0) & \\
\hline 3 & 113 & $(87.6)$ & 64 & $(94.1)$ & 49 & $(80.3)$ & \\
\hline Histological type & & & & & & & 0.48 \\
\hline Ductal & 120 & $(93.0)$ & 65 & (95.6) & 55 & $(90.2)$ & \\
\hline Lobular & 3 & (2.3) & 1 & (1.5) & 2 & (3.3) & \\
\hline Other & 6 & $(4.7)$ & 2 & (2.9) & 4 & $(6.6)$ & \\
\hline Axillary nodal status & & & & & & & 0.047 \\
\hline$\leq 3$ & 97 & $(75.2)$ & 56 & $(82.4)$ & 41 & $(67.2)$ & \\
\hline$>3$ & 32 & $(24.8)$ & 12 & $(17.6)$ & 20 & $(32.8)$ & \\
\hline Type of surgery & & & & & & & 0.53 \\
\hline Breast conserving & 43 & (33.3) & 21 & $(30.9)$ & 22 & $(36.1)$ & \\
\hline Mastectomy & 86 & $(66.7)$ & 47 & $(69.1)$ & 39 & $(63.9)$ & \\
\hline
\end{tabular}




\begin{tabular}{|lccccccc|}
\hline Characteristic & Total & & $\begin{array}{l}\text { Patients with a } \\
\text { BRCA7-like profile }\end{array}$ & $\begin{array}{l}\text { Patients with a non- } \\
\text { BRCA7-like profile }\end{array}$ & $\begin{array}{l}\text { P } \\
\text { value }\end{array}$ \\
\hline Axillary surgery & & & & & & & \\
\hline Dissection & 111 & $(86.0)$ & 54 & $(79.4)$ & 57 & $(93.4)$ & \\
\hline Sentinel node biopsy & 18 & $(14.0)$ & 14 & $(20.6)$ & 4 & $(6.6)$ & \\
\hline Treatment & & & & & & & \\
\hline T+ CEF & 69 & $(53.5)$ & 37 & $(54.4)$ & 32 & $(52.5)$ & \\
\hline TX+CEX & 60 & $(46.5)$ & 31 & $(45.6)$ & 29 & $(47.5)$ & \\
\hline
\end{tabular}

$\mathrm{P}$ values: patients with a $B R C A$ 7-like profile were compared with patients with a non-BRCA7-like profile. $\mathrm{P}$ values were calculated using Fisher's exact, Chi-square or linear-by-linear tests for categorical variables and Mann-Whitney U tests for continuous variables.

TNBC: triple negative breast cancer; $B R C A 7$-like: $B R C A 7$-like profile based on low coverage whole genome DNA next generation sequencing (ICNGS). Non-BRCA7-like: no BRCA7-like profile based on IcNGS. IQR: interquartile range; WHO: World Health Organization; T + CEF: 3 cycles of docetaxel 3-weekly, followed by 3 cycles of cyclophosphamide, epirubicin, and fluorouracil, 3-weekly; TX + CEX: 3 cycles of capecitabine plus docetaxel 3-weekly, followed by 3 cycles of cyclophosphamide, epirubicin and capecitabine, 3weekly.

\section{Association of BRCA1-like status with survival}

The median follow-up was 10.7 years for all 129 patients, with 35 recurrences and 32 deaths, and with a total person time of 1,085 years. In this cohort, BRCA7-like status was not significantly associated with prognosis: unadjusted hazard rates (HRs) of RFS and OS for BRCA7-like patients when compared to non-

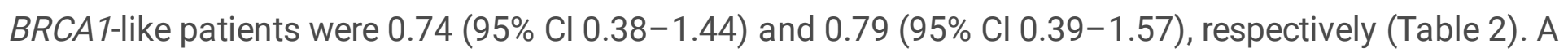
high number $(>3)$ of positive lymph nodes was significantly associated with an unfavorable RFS (HR 2.13; 95\% Cl 1.07-4.22), whereas T-stage (pT3 versus $\mathrm{pT} 1$ or $\mathrm{pT} 2$ : HR 1.90; 95\% $\mathrm{Cl} 0.67-5.39$ ) and histological grade was not (grade 3 versus grade 1 or 2 : HR $0.79 ; 95 \% \mathrm{Cl} 0.31-2.04$ ). 
Table 2

Cox proportional hazards analyses of prognostic and predictive value of BRCA7-like status for RFS and OS

\begin{tabular}{|c|c|c|c|c|c|c|c|c|}
\hline & RFS & & & & os & & & \\
\hline Variable & $\begin{array}{l}\text { No. Events/ } \\
\text { No. Patients }\end{array}$ & $H R^{*}$ & $\begin{array}{l}95 \% \\
\mathrm{Cl}^{*}\end{array}$ & $\begin{array}{l}P \\
\text { value }^{x}\end{array}$ & $\begin{array}{l}\text { No. Events/ } \\
\text { No. } \\
\text { Patients }\end{array}$ & $H R^{*}$ & $\begin{array}{l}95 \% \\
\mathrm{Cl}^{*}\end{array}$ & $\begin{array}{l}P \\
\text { value }^{*}\end{array}$ \\
\hline \multicolumn{9}{|l|}{$\begin{array}{l}\text { DNA-based } \\
\text { CNV pattern }\end{array}$} \\
\hline $\begin{array}{l}\text { Non-BRCA1- } \\
\text { like }\end{array}$ & $19 / 61$ & 1 & & & $17 / 61$ & 1 & & \\
\hline BRCA1-like & 16/68】 & $0.74 \rrbracket$ & $\begin{array}{l}0.38- \\
1.44 \rrbracket\end{array}$ & $0.37 \rrbracket$ & 15/68》 & $0.79 \rrbracket$ & $\begin{array}{l}0.39- \\
1.57 \square\end{array}$ & $0.49 \rrbracket$ \\
\hline \multicolumn{9}{|l|}{$\begin{array}{l}\text { BRCA1-like } \\
\text { tumors }\end{array}$} \\
\hline $\mathrm{T}+\mathrm{CEF}$ & $10 / 37$ & 1 & & & $9 / 37$ & 1 & & \\
\hline TX + CEX & $6 / 310$ & $0.66 \rrbracket$ & $\begin{array}{l}0.24- \\
1.81 \rrbracket\end{array}$ & $0.42 \rrbracket$ & 6/31区 & $0.75 \rrbracket$ & $\begin{array}{l}0.27- \\
2.11 \rrbracket\end{array}$ & $0.59 \rrbracket$ \\
\hline \multicolumn{9}{|l|}{$\begin{array}{l}\text { Non-BRCA1- } \\
\text { like tumors }\end{array}$} \\
\hline $\mathrm{T}+\mathrm{CEF}$ & $15 / 32$ & 1 & & & $14 / 32$ & 1 & & \\
\hline TX + CEX & $4 / 29 \rrbracket$ & $0.23 \rrbracket$ & $\begin{array}{l}0.08- \\
0.70 \rrbracket\end{array}$ & $<$ & $3 / 29 \rrbracket$ & $0.19 \rrbracket$ & $\begin{array}{l}0.05- \\
0.66 \text { \ }\end{array}$ & $<$ \\
\hline
\end{tabular}

Interaction test between $B R C A 7$-like status and chemotherapy regimen: $\otimes \mathrm{P}=0.17 ; \otimes \mathrm{P}=0.09 .{ }^{*} \mathrm{All}$ Cox proportional hazard analyses shown here were unadjusted for clinic-pathological variables. Similar results were obtained when adjusted for one covariate at the time (due to the relative small number of events).

RFS: recurrence-free survival; OS: overall survival; HR: hazard ratio; Cl: confidence interval; CNV: copy number variation; T + CEF: 3 cycles of docetaxel 3-weekly, followed by 3 cycles of cyclophosphamide, epirubicin, and fluorouracil, 3-weekly; TX + CEX: 3 cycles of capecitabine plus docetaxel 3-weekly, followed by 3 cycles of cyclophosphamide, epirubicin and capecitabine, 3-weekly.

\section{Association of BRCA1-like status with benefit of adjuvant capecitabine-containing chemotherapy}

Overall, adjuvant capecitabine-containing chemotherapy (TX + CEX) was more effective than the conventional chemotherapy ( $T+C E F$ ) in our cohort of 129 TNBC patients (RFS: HR 0.39 ; $95 \% \mathrm{Cl} 0.19-$ $0.82 ; \mathrm{P}=0.01$ ). This is in line with the treatment effect in all 202 TNBC cases of the FinXX trial (RFS: HR $0.54 ; 95 \% \mathrm{Cl} 0.31-0.92 ; \mathrm{P}=0.02$ ) [28]. While in non- $B R C A 7$-like patients adjuvant capecitabine-containing 
chemotherapy was significantly more effective than conventional chemotherapy (RFS: HR $0.23 ; 95 \% \mathrm{Cl}$ $0.08-0.70 ; \mathrm{P}<0.01$ ), this was not observed in patients with a BRCA1-like tumor (RFS: HR $0.66 ; 95 \% \mathrm{Cl}$ 0.24-1.81; $P=0.42$ ). However, the beneficial effect of the adjuvant capecitabine-containing regimen did not differ by $B R C A 1$-like status (P interaction $=0.17)($ Table 2, Fig. 2). Similar results were obtained after adjustment for each of the clinico-pathological variables ( $P$-values ranging from 0.15 to 0.22 ).

Furthermore, similar results were observed for OS ( $P$ interaction $=0.09)$ (Table 2, Additional file 3: Figure S1).

\section{DNA-based CNV BRCA1-like status versus RNA-based NanoString BRCAness signature}

Both scores of DNA-based CNV BRCA7-like classifier and the RNA-based NanoString BRCAness signature were available for 103/202 TNBC patients (Fig. 3). The established cut-off for the BRCA1-like classifier $(0.63)$ occurred at the 42.7 th percentile in this dataset. The BRCAness signature score does not have an established cut-off. We therefore dichotomized at the 42.7th percentile of the ranked BRCAness scores (6.18). For 78.6\% (81/103) there is concordance in BRCA7-like/BRCAness classification. The $21.4 \%$ $(22 / 103)$ disagreement is equal in both directions and the discordant patients are at an intermediate risk of recurrence between the concordant patients.

\section{Discussion}

In the present study we found no evidence that benefit from adjuvant capecitabine-containing chemotherapy compared to conventional chemotherapy is associated with BRCA1-like status in earlystage TNBC patients. Therefore, it is unlikely that the BRCA1-like classifier will help in selecting patients for adjuvant capecitabine-enriched chemotherapy.

Our hypothesis of differential treatment effects was based on previous observations made by Van Rossum et al. [26]. Their retrospective analysis of the randomized GAIN study suggested that patients with BRCA7-like tumors have a selective treatment benefit from a conventional dose-dense chemotherapy regimen, containing capecitabine, if compared with non-myeloablative, intensified dose-dense chemotherapy. There are three important differences between the GAIN study and the FinXX study. The main difference is that the FinXX study compared two regimens that only differed by the addition of capecitabine (yes/no) and a slightly lower dose of docetaxel in the capecitabine arm [27], while the GAIN study compared miscellaneous regimens that differed in drug dose and drug combinations [35]. Second, the GAIN study accrued only node-positive patients, while the FinXX study also recruited high risk nodenegative patients. And lastly, the capecitabine dose and schedule differed between the studies (10 cycles of capecitabine $1000 \mathrm{mg} / \mathrm{m}^{2}$ twice daily administered on day 1-14 in a three weekly schedule in the GAIN trial versus 6 cycles of capecitabine $900 \mathrm{mg} / \mathrm{m}^{2}$ twice daily on day $1-15$ every 3 weeks in the FinXX study). Interestingly, we observed a significant capecitabine benefit for the non-BRCA7-like group (RFS and OS P value $<0.01$ ) and less evidence of benefit for the BRCA1-like group (RFS P value 0.42; OS P value 0.59), in contrast to the observations of Van Rossum et al. [26]. Whether or not these two observations represent inconsistent patterns is difficult to determine due to the small sample size in these 
unplanned subgroup analyses. The patterns are actually reversed, so unless the direction of effects changes with more data, narrower confidence intervals will only make both studies less consistent with each other.

Two biological processes may explain why BRCA7-like status is not predictive for benefit of adjuvant capecitabine treatment. First, although capecitabine causes DNA-damage [5], it may not specifically result in DNA damage that is dependent on a proficient homologous recombination machinery resulting in errorfree DNA repair [36]. In fact, capecitabine and its active form 5-fluorouracil lead to (1) DNA base pair mismatches which are repaired by the DNA mismatch repair (MMR) pathway [37] and (2) inhibition of DNA replication, leading to abasic sites that are repaired by base excision repair (BER) proteins [38]. Second, there are some data suggesting that capecitabine might result in better outcome in TNBC patients with grade 2 tumors, but not grade 3 [39]. Since BRCA7-like tumors are enriched for high-grade tumors, our findings of no predictive value of $B R C A 7$-like status for benefit of capecitabine support these observations.

Our results are in line with previous preclinical findings of Quinn et al who observed no differential doseresponse effect of capecitabine in BRCA1-mutated compared with wild-type BRCA1 human BC cells [40], and with Alli et al who found a 5-fold lower sensitivity to 5-fluoro-uracil of BRCA7-deficient compared to wild-type BRCA1 murine mammary epithelial cells [41]. Moreover, our findings are consistent with the recent observations of Asleh et al. [11]. In the same dataset, they found no significant association of improved outcome and signatures associated with DNA damage repair, including HRD and BRCAness, using an 800-gene panel in 111 early-stage TNBC patients treated with adjuvant capecitabine in the FinXX trial. Additionally, we demonstrated $78.6 \%$ concordance between our DNA-based CNV BRCA7-like classifier and the RNA-based NanoString BRCAness signature used by Asleh et al. [11]. Taken together, these data indicate that benefit of adjuvant capecitabine-containing chemotherapy could not be predicted by deficiency in homologous recombination. Currently, the NordicTrip (ClinicalTrials.gov Identifier: NCT04335669) is an ongoing translational clinical trial in early-stage TNBC prospectively comparing the effect on pathologic complete response ( $\mathrm{pCR}$ ) rate of adding capecitabine to epirubicin plus cyclophosphamide followed by carboplatin plus paclitaxel neoadjuvant chemotherapy, stratified for HRD positive versus HRD negative/ HRD-intermediate. Results of this study have to be awaited to further clarify the value of HRD as a predictive biomarker for benefit of capecitabine-containing chemotherapy in early-stage TNBC.

The main strength of our study is the study design, i.e., a prospective, randomized controlled trial with collection of archival material. This prospective-retrospective design is the first choice to assess a putative predictive biomarker in case a prospective randomized clinical trial is not feasible, because such trials require huge numbers of patients, are costly and take many years to complete [42]. An additional strength, in contrast to the exploratory analyses of Asleh et al. using an RNA 800-gene panel without predefined cutoff for the BRCAness signature [11], is that we evaluated a single biomarker with a predefined cutoff based on prior biological and empirical evidence [21, 23-25]. This confirmatory objective is required to establish the implementation of a predictive biomarker or to refute it [42]. 
A limitation of the present study is the small sample size, which is due to the fact that the FinXX trial was powered to evaluate the main effect of capecitabine among patients with any biological type of breast cancer rather than a treatment-marker interaction in the subgroup of TNBC patients. In addition, the number of patients was further reduced by the failure to obtain BRCA7-like status for all TNBC patients for several reasons. However, our patient group accounts for 129 (63.9\%) of the 202 accrued TNBC patient in the FinXX trial, which is within the recommended range of sample size for a study to evaluate predictive biomarkers [42]. Furthermore, the included patients did not differ meaningfully from all accrued TNBC patients for the evaluated clinical variables. Lastly, whether BRCA1-like status has predictive value for capecitabine-containing chemotherapy only in individuals with residual disease after neoadjuvant chemotherapy is still an open question. Collaborate efforts to assess this in the CREATE-X trial are ongoing.

Our findings may have implications for treatment decisions in early-stage TNBC patients. Currently, the addition of the polyadenosine 5'diphosphoribose polymerase (PARP) inhibitors in the (neo) adjuvant treatment of these TNBC patients is an emerging area of investigation [43-46]. The OlympiA trial is one very important and large trial that evaluates the efficacy of adjuvant treatment with olaparib, a PARP inhibitor, compared to placebo in patients with non-metastatic, germline $B R C A$ mutated ( $g B R C A m)$ high risk HER2-negative primary breast cancer (ClinicalTrials.gov Identifier: NCT02032823). An unanswered question remains what the added value of adjuvant treatment with PARP inhibitors would be compared to the current clinical practice of capecitabine-containing treatment. In our present study, we demonstrate that the efficacy of adjuvant capecitabine treatment in early-stage TNBC patients does not differ by $B R C A 7$-like status. Therefore, the added value of PARP inhibition to capecitabine-containing adjuvant chemotherapy in gBRCAm-associated or BRCA7-like TNBC patients that did not achieve a pathological complete remission on standard neoadjuvant chemotherapy needs to be investigated.

\section{Conclusions}

Our data suggests that benefit from adjuvant capecitabine added to standard adjuvant chemotherapy in early-stage TNBC patients does not depend on BRCA7-like status. Identifying biomarkers that define the subgroup of early-stage TNBC patients who benefit from adjuvant capecitabine remains an urgent need in future research.

\section{Abbreviations}

(a)CGH: (array) comparative genomic hybridization; BER: base excision repair; CNV: copy number variation; DFS: disease-free survival; ER: estrogen receptor; FFPE: formalin-fixed paraffin-embedded; HRD: homologous recombination deficiency; MMR: mismatch repair; OS: overall survival; PARP: polyadenosine 5'diphosphoribose polymerase; pCR: pathologic complete response; PR: progesterone receptor; RFS: recurrence-free survival; SRA: Sequence Read Archive; TNBC: triple negative breast cancer; T+CEF: 3 cycles of docetaxel $80 \mathrm{mg} / \mathrm{m}^{2}$ 3-weekly, followed by 3 cycles of cyclophosphamide $600 \mathrm{mg} / \mathrm{m}^{2}$, epirubicin $75 \mathrm{mg} / \mathrm{m}^{2}$, and fluorouracil $600 \mathrm{mg} / \mathrm{m}^{2}$, 3-weekly; TX+CEX: 3 cycles of capecitabine 900 
$\mathrm{mg} / \mathrm{m}^{2}$ twice daily plus docetaxel $60 \mathrm{mg} / \mathrm{m}^{2}$ 3-weekly, followed by 3 cycles of cyclophosphamide 600 $\mathrm{mg} / \mathrm{m}^{2}$, epirubicin $75 \mathrm{mg} / \mathrm{m}^{2}$ and capecitabine $900 \mathrm{mg} / \mathrm{m}^{2}$ twice daily, 3-weekly; WHO: World Health Organization; $\mathrm{X}$ : capecitabine

\section{Declarations}

\section{Ethics approval and consent to participate}

The study was approved by the Ethics Committee of the participating medical institutions and the National Agency for Medicines, Finland. Patients supplied written informed consent to allow the use of their tumor tissue for clinical study related research purposes. The Institutional Review Boards at Helsinki University, Finland, approved the use of archival tissue for the current translational study.

\section{Consent for publication}

Not Applicable.

\section{Availability of data and materials}

The clinical datasets used and analyzed during the current study are available from Heikki Joensuu on reasonable request. The sequencing datasets generated and analyzed during the current study are available in the SRA repository, [https://www.ncbi.nlm.nih.gov/Traces/study1/?acc=PRJNA647428\&].

\section{Competing interests}

HJ has ownership interest (including patents) at Sartar Therapeutics and is a board member, has a coappointment at Orion Pharma and is employed by Orion Pharma, and has received fees from Neutron Therapeutics. SCL reports grants from ZonMw and A Sister's Hope during the conduct of the study. SCL is an advisory board member for AstraZeneca, Cergentis, IBM, Pfizer and Roche, and received institutional research grants from Agendia, AstraZeneca, Eurocept-pharmaceuticals and Pfizer. In addition, SCL received institutional research grants and institutional non-financial support from Genentech, Novartis, Roche, Tesaro and Immunomedics and other institutional support from AstraZeneca, Pfizer, Cergentis, Daiichi Sankyo, IBM and Bayer outside the submitted work. MK is an advisory board member for BMS, Roche, MSD and Daiichi and received institutional research support from AstraZeneca, BMS and Roche outside the submitted work.

The other authors declare that they have no competing interests.

\section{Funding}

This work was supported by the Dutch Cancer Society (Project Number 10603). The funding source had no role in study design, data collection and analyses, decision to publish, or preparation of the manuscript. 


\section{Authors' contributions}

LWB performed the data acquisition and statistical analysis, interpreted the data, and drafted the manuscript. KJ performed statistical analyses, interpreted the data and critically revised the manuscript. HJ and SL performed the data acquisition and reviewed the manuscript. CS, WB, PCS and RJCK generated the sequencing data and critically revised the manuscript. MO and PMN were involved in acquiring the BRCA7-like status, interpreting the data, and critically revised the manuscript. $\mathrm{HL}$ and $\mathrm{MK}$ critically revised the manuscript. MH designed the study, analyzed and interpreted the data and critically revised the manuscript. SCL designed the study, interpreted the data, and critically revised the manuscript.

All authors read and approved the final manuscript.

\section{Acknowledgments}

The authors would like to thank all patients for participating in the study.

\section{References}

1. Dent R, Trudeau M, Pritchard KI, Hanna WM, Kahn HK, Sawka CA, et al. Triple-negative breast cancer: clinical features and patterns of recurrence. Clin Cancer Res. 2007;13(15 Pt 1):4429-34.

2. Hudis CA, Gianni L. Triple-negative breast cancer: an unmet medical need. Oncologist. 2011;16(Suppl 1):1-11.

3. Schmid P, Cortes J, Pusztai L, McArthur H, Kümmel S, Bergh J, et al. Pembrolizumab for Early TripleNegative Breast Cancer. N Engl J Med. 2020;382(9):810-21.

4. Nanda R, Liu MC, Yau C, Shatsky R, Pusztai L, Wallace A, et al. Effect of Pembrolizumab Plus Neoadjuvant Chemotherapy on Pathologic Complete Response in Women With Early-Stage Breast Cancer: An Analysis of the Ongoing Phase 2 Adaptively Randomized I-SPY2 Trial. JAMA Oncology. 2020;6(5):676-84.

5. Longley DB, Harkin DP, Johnston PG. 5-fluorouracil: mechanisms of action and clinical strategies. Nat Rev Cancer. 2003;3(5):330-8.

6. van Mackelenbergh M, Seither F, Möbus V, O'Shaugnessy J, Martin M, Joensuu H, et al. Abstract GS107: Effects of capecitabine as part of neo-/adjuvant chemotherapy. A meta-analysis of individual patient data from 12 randomized trials including 15,457 patients. Cancer Res. 2020, 80(4 Supplement):GS1-07-GS1-07.

7. Masuda N, Lee SJ, Ohtani S, Im YH, Lee ES, Yokota I, et al. Adjuvant Capecitabine for Breast Cancer after Preoperative Chemotherapy. N Engl J Med. 2017;376(22):2147-59.

8. Burstein HJ, Curigliano G, Loibl S, Dubsky P, Gnant M, Poortmans P, et al. Estimating the benefits of therapy for early-stage breast cancer: the St. Gallen International Consensus Guidelines for the primary therapy of early breast cancer 2019. Ann Oncol. 2019;30(10):1541-57. 
9. Denduluri N, Chavez-MacGregor M, Telli ML, Eisen A, Graff SL, Hassett MJ, et al. Selection of Optimal Adjuvant Chemotherapy and Targeted Therapy for Early Breast Cancer: ASCO Clinical Practice Guideline Focused Update. J Clin Oncol. 2018;36(23):2433-43.

10. Cardoso F, Kyriakides S, Ohno S, Penault-Llorca F, Poortmans P, Rubio IT, et al. Early breast cancer: ESMO Clinical Practice Guidelines for diagnosis, treatment and follow-upt. Ann Oncol. 2019;30(8):1194-220.

11. Asleh K, Brauer HA, Sullivan A, Lauttia S, Lindman H, Nielsen TO, et al. Predictive Biomarkers for Adjuvant Capecitabine Benefit in Early-Stage Triple-Negative Breast Cancer in the FinXX Clinical Trial. Clin Cancer Res. 2020;26(11):2603-14.

12. Lord CJ, Ashworth A. BRCAness revisited. Nat Rev Cancer. 2016;16(2):110-20.

13. Brok WDd, Schrader KA, Sun S, Tinker AV, Zhao EY, Aparicio S, et al. Homologous Recombination Deficiency in Breast Cancer: A Clinical Review. 2017;(1):1-13.

14. Couch FJ, Hart SN, Sharma P, Toland AE, Wang X, Miron P, et al. Inherited mutations in 17 breast cancer susceptibility genes among a large triple-negative breast cancer cohort unselected for family history of breast cancer. J Clin Oncol. 2015;33(4):304-11.

15. Robertson L, Hanson H, Seal S, Warren-Perry M, Hughes D, Howell I, et al. BRCA1 testing should be offered to individuals with triple-negative breast cancer diagnosed below 50 years. Br $\mathrm{J}$ Cancer. 2012;106(6):1234-38.

16. Hartman A-R, Kaldate RR, Sailer LM, Painter L, Grier CE, Endsley RR, et al. Prevalence of BRCA mutations in an unselected population of triple-negative breast cancer. Cancer. 2012;118(11):278795.

17. Gonzalez-Angulo AM, Timms KM, Liu S, Chen H, Litton JK, Potter J, et al. Incidence and outcome of BRCA mutations in unselected patients with triple receptor-negative breast cancer. Clin Cancer Res. 2011;17(5):1082-89.

18. Rummel S, Varner E, Shriver CD, Ellsworth RE. Evaluation of BRCA1 mutations in an unselected patient population with triple-negative breast cancer. Breast Cancer Res Treat. 2013;137(1):119-25.

19. Turner N, Tutt A, Ashworth A. Hallmarks of 'BRCAness' in sporadic cancers. Nat Rev Cancer. 2004;4(10):814-9.

20. Tung NM, Garber JE. BRCA1/2 testing: therapeutic implications for breast cancer management. Br J Cancer. 2018;119(2):141-52.

21. Joosse SA, van Beers EH, Tielen IH, Horlings H, Peterse JL, Hoogerbrugge $N$, et al. Prediction of BRCA1-association in hereditary non-BRCA1/2 breast carcinomas with array-CGH. Breast Cancer Res Treat. 2009;116(3):479-89.

22. Vollebergh MA, Lips EH, Nederlof PM, Wessels LF, Schmidt MK, van Beers EH, et al. An aCGH classifier derived from BRCA1-mutated breast cancer and benefit of high-dose platinum-based chemotherapy in HER2-negative breast cancer patients. Ann Oncol. 2011;22(7):1561-70.

23. Vollebergh MA, Lips EH, Nederlof PM, Wessels LF, Wesseling J, Vd Vijver MJ, et al. Genomic patterns resembling BRCA1- and BRCA2-mutated breast cancers predict benefit of intensified carboplatin- 
based chemotherapy. Breast Cancer Res. 2014;16(3):R47.

24. Schouten PC, Marme F, Aulmann S, Sinn HP, van Essen HF, Ylstra B, et al. Breast cancers with a BRCA1-like DNA copy number profile recur less often than expected after high-dose alkylating chemotherapy. Clin Cancer Res. 2015;21(4):763-70.

25. Schouten PC, Gluz O, Harbeck N, Mohrmann S, Diallo-Danebrock R, Pelz E, et al. BRCA1-like profile predicts benefit of tandem high dose epirubicin-cyclophospamide-thiotepa in high risk breast cancer patients randomized in the WSG-AM01 trial. Int J Cancer. 2016;139(4):882-89.

26. van Rossum AGJ, Schouten PC, Weber KE, Nekljudova V, Denkert C, Solbach C, et al. BRCA1-like profile is not significantly associated with survival benefit of non-myeloablative intensified chemotherapy in the GAIN randomized controlled trial. Breast Cancer Res Treat. 2017;166(3):77585.

27. Joensuu H, Kellokumpu-Lehtinen PL, Huovinen R, Jukkola-Vuorinen A, Tanner M, Kokko R, et al. Adjuvant capecitabine, docetaxel, cyclophosphamide, and epirubicin for early breast cancer: final analysis of the randomized FinXX trial. J Clin Oncol. 2012;30(1):11-8.

28. Joensuu H, Kellokumpu-Lehtinen PL, Huovinen R, Jukkola-Vuorinen A, Tanner M, Kokko R, et al. Adjuvant Capecitabine in Combination With Docetaxel, Epirubicin, and Cyclophosphamide for Early Breast Cancer: The Randomized Clinical FinXX Trial. JAMA Oncol. 2017;3(6):793-800.

29. Li H. Aligning sequence reads, clone sequences and assembly contigs with BWA-MEM. ArXiv. 2013, 1303.

30. Quinlan AR, Hall IM. BEDTools: a flexible suite of utilities for comparing genomic features. Bioinformatics. 2010;26(6):841-42.

31. Amemiya HM, Kundaje A, Boyle AP. The ENCODE Blacklist: Identification of Problematic Regions of the Genome. Sci Rep. 2019;9(1):9354.

32. Sequence Read Archive (SRA). BioProject number PRJNA647428. https://wwwncbinlmnihgov/Traces/study1/?acc=PRJNA647428\&.

33. Schouten PC, Grigoriadis A, Kuilman T, Mirza H, Watkins JA, Cooke SA, et al. Robust BRCA1-like classification of copy number profiles of samples repeated across different datasets and platforms. Mol Oncol. 2015;9(7):1274-86.

34. Brauer HA, Mashadi-Hossein A, Buckingham W, Danaher P, Ferree S. Gene expression signature development to decode breast cancer heterogeneity. J Clin Oncol. 2018;36(15_suppl):e24243-e43.

35. Möbus V, von Minckwitz G, Jackisch C, Lück HJ, Schneeweiss A, Tesch H, et al. German Adjuvant Intergroup Node-positive Study (GAIN): a phase III trial comparing two dose-dense regimens (iddEPC versus ddEC-PwX) in high-risk early breast cancer patients. Ann Oncol. 2017;28(8):1803-10.

36. Xeloda $150 \mathrm{mg}$ and $500 \mathrm{mg}$ Film-Coated Tablets - summary of product characteristics (SPC). (eMC). https://www.medicines.org.uk/emc/medicine/4619/SPC/Xeloda+150mg+and+500mg+Filmcoated+Tablets/. Assessed 2 December 2020.

37. Iwaizumi M, Tseng-Rogenski S, Carethers JM. DNA mismatch repair proficiency executing 5fluorouracil cytotoxicity in colorectal cancer cells. Cancer Biol Ther. 2011;12(8):756-64. 
38. Huehls AM, Huntoon CJ, Joshi PM, Baehr CA, Wagner JM, Wang X, et al. Genomically Incorporated 5Fluorouracil that Escapes UNG-Initiated Base Excision Repair Blocks DNA Replication and Activates Homologous Recombination. Mol Pharmacol. 2016;89(1):53-62.

39. O'Shaughnessy J, Koeppen H, Xiao Y, Lackner MR, Paul D, Stokoe C, et al. Patients with Slowly Proliferative Early Breast Cancer Have Low Five-Year Recurrence Rates in a Phase III Adjuvant Trial of Capecitabine. Clin Cancer Res. 2015;21(19):4305-11.

40. Quinn JE, Kennedy RD, Mullan PB, Gilmore PM, Carty M, Johnston PG, et al. BRCA1 Functions as a Differential Modulator of Chemotherapy-induced Apoptosis. Cancer Res. 2003;63(19):6221-28.

41. Alli E, Sharma VB, Hartman AR, Lin PS, McPherson L, Ford JM. Enhanced sensitivity to cisplatin and gemcitabine in Brca1-deficient murine mammary epithelial cells. BMC Pharmacol. 2011;11:7.

42. Simon RM, Paik S, Hayes DF. Use of archived specimens in evaluation of prognostic and predictive biomarkers. J Natl Cancer Inst. 2009;101(21):1446-52.

43. Rugo HS, Olopade OI, DeMichele A, Yau C, van 't Veer LJ, Buxton MB, et al. Adaptive Randomization of Veliparib-Carboplatin Treatment in Breast Cancer. N Engl J Med. 2016;375(1):23-34.

44. Loibl S, O'Shaughnessy J, Untch M, Sikov WM, Rugo HS, McKee MD, et al. Addition of the PARP inhibitor veliparib plus carboplatin or carboplatin alone to standard neoadjuvant chemotherapy in triple-negative breast cancer (BrighTNess): a randomised, phase 3 trial. Lancet Oncol. 2018;19(4):497-509.

45. Fasching PA, Link T, Hauke J, Seither F, Jackisch C, Klare P, et al. Neoadjuvant paclitaxel/olaparib in comparison to paclitaxel/carboplatinum in patients with HER2-negative breast cancer and homologous recombination deficiency (GeparOLA study). Ann Oncol. 2020.

46. Severson TM, Wolf DM, Yau C, Peeters J, Wehkam D, Schouten PC, et al. The BRCA1ness signature is associated significantly with response to PARP inhibitor treatment versus control in the I-SPY 2 randomized neoadjuvant setting. Breast Cancer Res. 2017;19(1):99.

\section{Figures}




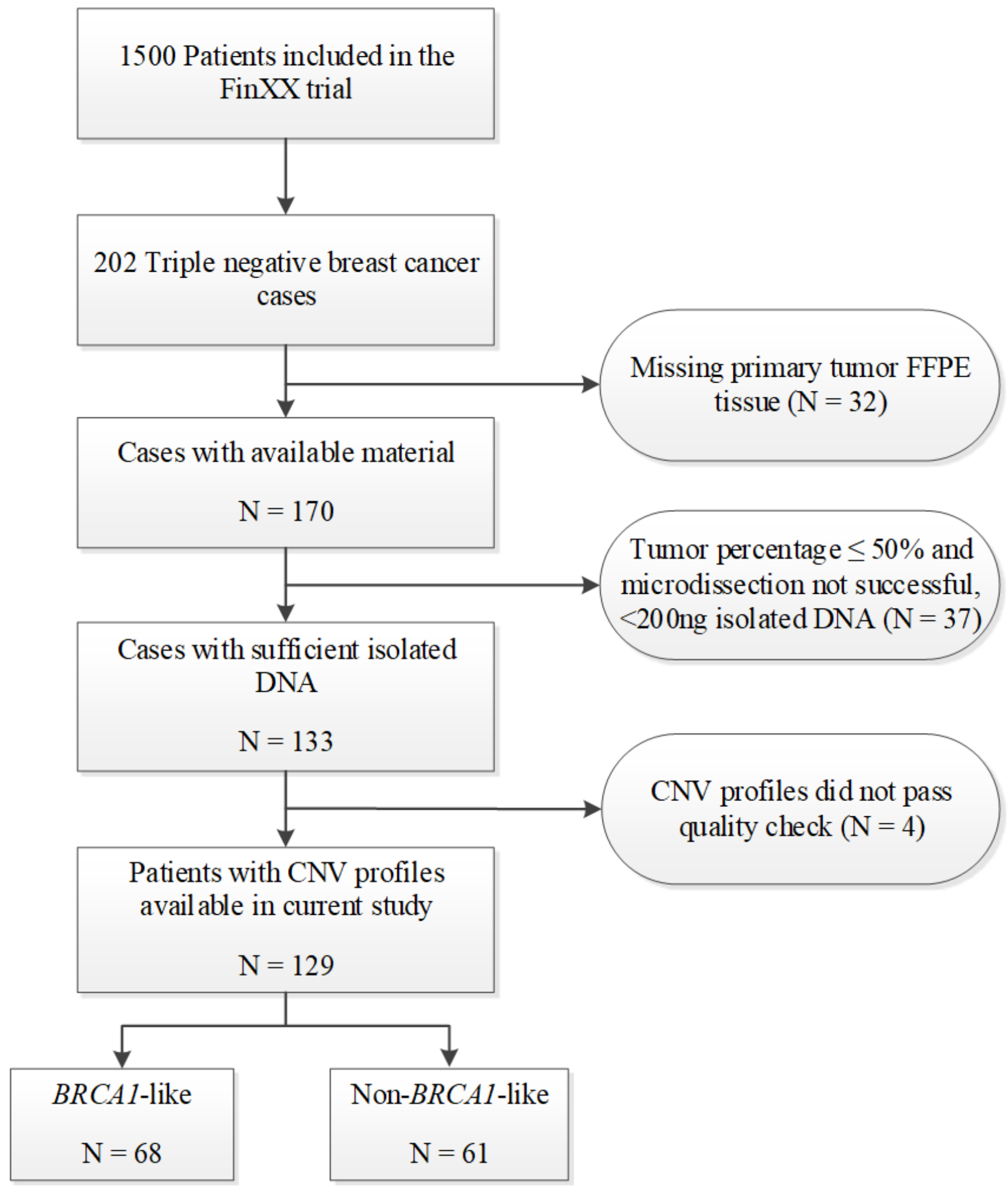

\section{Figure 1}

Flow diagram of patient selection in the current study Reasons for dropout are listed. Tumors of 129 patients could be evaluated for BRCA1-like status. Triple negative breast cancer was defined as estrogen (ER) and progesterone receptor (PR) negativity ( $<10 \%)$, and no HER2 overexpression. FFPE: formalin-fixed paraffin-embedded; CNV: copy number variation; BRCA1-like: BRCA1-like profile based on low coverage 
whole genome DNA next generation sequencing (ICNGS). Non-BRCA1-like: no BRCA1-like profile based on IcNGS.

A

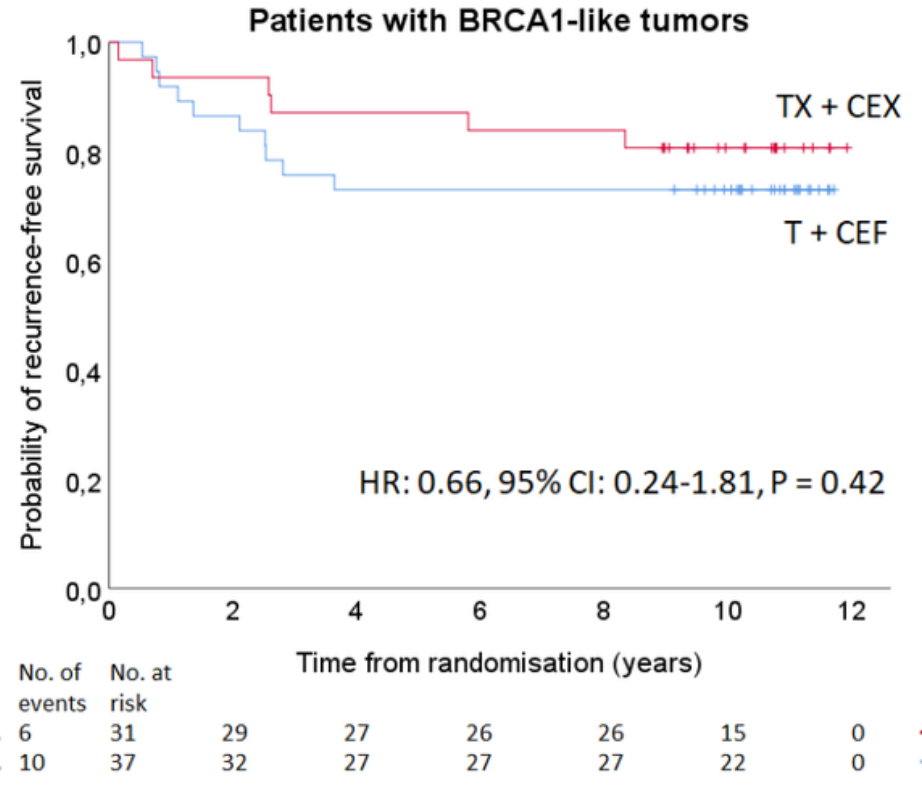

C

$\begin{array}{lr} & \begin{array}{r}\text { TX+CEX } \\ \text { No. Events } \\ \text { No. Patients }\end{array} \\ \text { BRCA-like status } & \\ \text { BRCA1-like tumor } & 6 / 31 \\ \text { non-BRCA1-like tumor } & 4 / 29 \\ \text { Overall } & \end{array}$

T+CEF
B

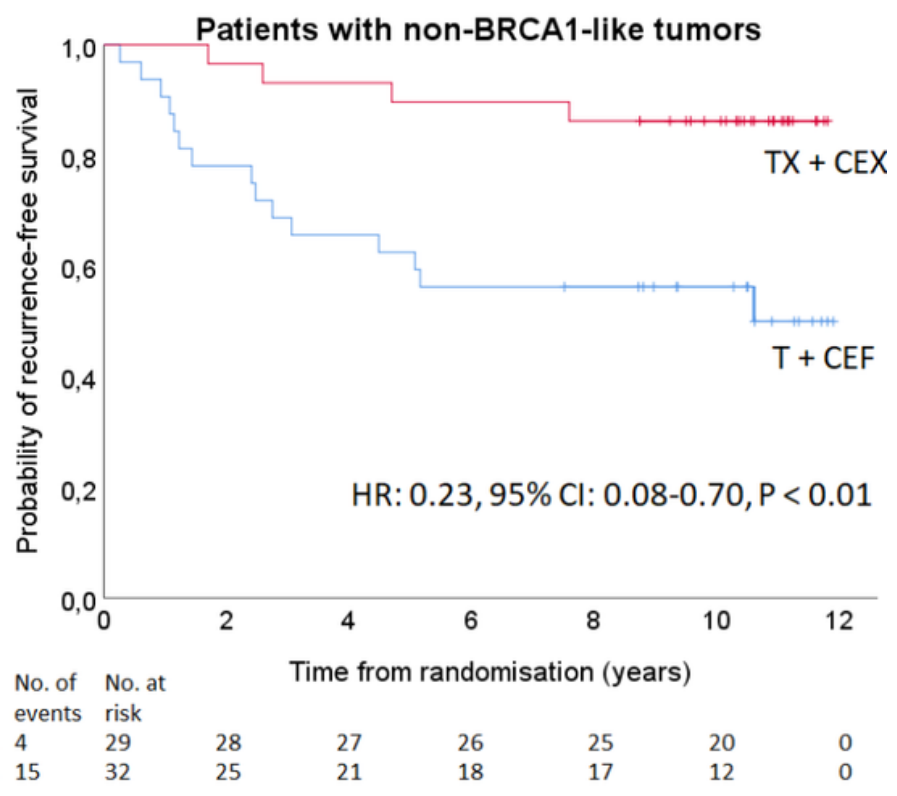

No. Events/

No. Patients

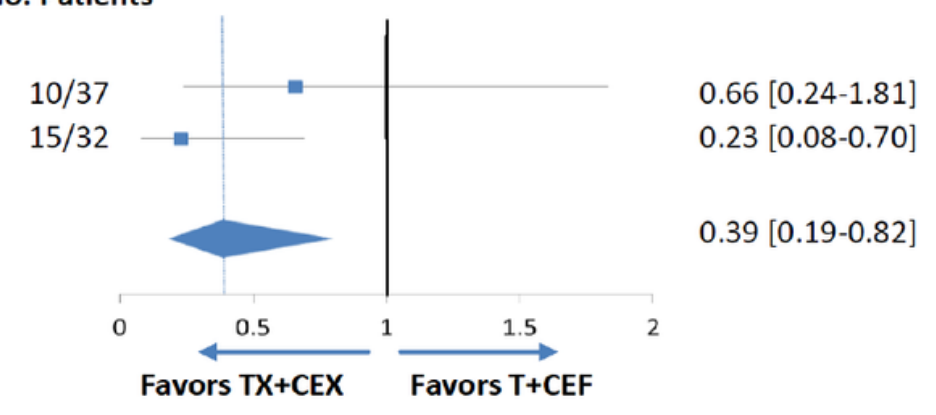

Figure 2

Recurrence-free survival for TNBC patients by BRCA1-like status and allocated adjuvant treatment Kaplan-Meier curves of RFS for TNBC patients with BRCA1-like (A) and non-BRCA1-like tumors (B) according to treatment. Number of events and patients at risk are reported below the figure. Unadjusted hazard ratios are derived from Cox regression models $(A+B)$. Similar results were obtained when HRs were adjusted for clinico-pathological variables. (C) Forest plot of hazard ratios for recurrence-free survival according to BRCA1-like status and treatment. Patients had been randomly assigned between adjuvant TX+CEX or T+CEF. HR: hazard ratio; Cl: confidence interval; RFS: recurrence free survival; TNBC: triple negative breast cancer; TX+CEX: 3 cycles of capecitabine plus docetaxel 3-weekly, followed by 3 cycles of cyclophosphamide, epirubicin and capecitabine, 3-weekly; T+CEF: 3 cycles of docetaxel 3weekly, followed by 3 cycles of cyclophosphamide, epirubicin, and fluorouracil, 3-weekly. 


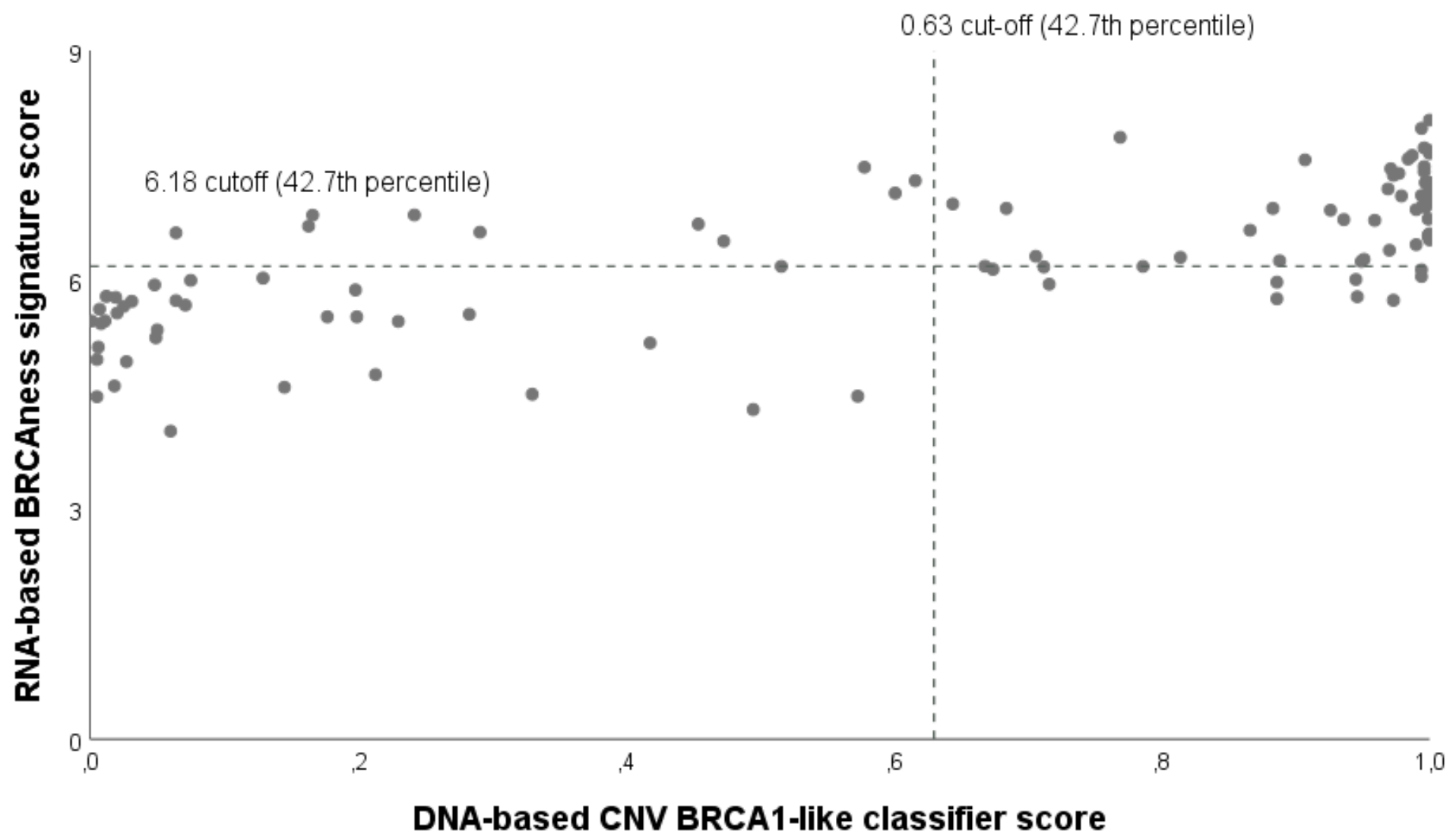

Figure 3

DNA-based CNV BRCA1-like classifier versus RNA-based BRCAness signature Scatterplot illustrating the corresponding DNA-based CNV BRCA1-like classifier score and RNA-based NanoString BRCAness signature score belonging to the same tumor. Both scores were available for 103/202 TNBC patients. The dashed lines illustrate the cut-off points when the scores are dichotomized at the percentile of the established cut-off for the BRCA1-like classifier (42.7th percentile).

\section{Supplementary Files}

This is a list of supplementary files associated with this preprint. Click to download.

- Additionalfile1.Supplementarymethods.docx

- Additionalfile2.TableS1.docx

- Additionalfile3.FigureS1.docx 